\title{
Evaluation of Choroidal Thickness after Uncomplicated Phacoemulsification Surgery Using Optical Coherence Tomography
}

E.E.Saber, A.A.h.Fayed, M.F.Farid and A.S.Salem

\author{
Ophthalmology, Dept., Faculty of Medicine, Benha Univ., Benha, Egypt
}

E-Mail:ayootabaskota88@gmail.com

\begin{abstract}
Phacoemulsification is an invasive procedure that has become the most common intraocular surgery and usually improves the visual outcome. We aimed to evaluate the potential changes in the subfoveal choroidal thickness (SFCT) after cataract surgery, using Enhanced Depth Imaging OCT. The study was applied on 30 eyes of 30 patients with no medical or ocular disorder and no history of previous ocular surgery . 15 males and 15 females, divided into two groups : group A (15 eyes) - underwent cataract surgery without using non-steroidal anti-inflammatory eye drop before surgery. group B (15 eyes) - will underwent cataract surgery with the use of non-steroidal anti-inflammatory eye drop 3 times ( one drop every 20 mins for hour ) just before surgery and screened to meet the inclusion criteria. Each patient was examined by EDI-OCT four times, one of them done in the day before surgery and three others after surgery in D7, M1 and M3. Our results confirm slight increases in macular thickness start 7 days after surgery and the lastest was one month after surgery. These changes in macular thickness eventually return normally near 3 months after surgery. There was no significant difference in B-group which administered NSAIDs before surgery. Uncomplicated phacoemulsification induces nonpathologic increases in retinal macular thickness probably due to the inflammatory insult of the surgery; however these changes are not accompanied by significant changes in choroidal thickness.
\end{abstract}

Keywords: Choroidal Thickness, Phacoemulsification, Optical Coherence Tomography.

\section{Introduction}

The pathogenesis of macular edema is considered as a result of releases inflammatory mediators, such as prostaglandins, leukotrienes, and cytokines, which leads to the breakdown of the blood-aqueous barrier and of the blood-retinal barrier [1].

The choroid may also be involved in the inflammatory response after cataract surgery. However, its contribution to the development of (PCME) is hypothetical and needs to be investigated [2].

Optical coherence tomography is a high-resolution, cross-sectional imaging technique that allows detailed assessment of retinal thickness and morphologic evaluation of the retinal layers. OCT imaging has rapidly been developed over the past few years and has been integrated into routine ophthalmic clinical practice and trials. It has become an important clinical and research tool for the diagnosis, follow up, and assessment of new treatment modalities for many diseases that affect the posterior pole of the eye [3].

Spectral-domain optical coherence tomography (SDOCT) provides detailed histological images of the retina and provide information for diagnosis and follow up. The other method for visualizing the choroid is enhanced depth imaging OCT (EDI-OCT). Enhanced depth imaging OCT is an innovation of post processing that uses a commercially available spectral-domain OCT device. Deep choroidal images are enhanced by taking inverted images and using multiple B-scan averaging to improve the signal-to noise ratio (SNR) [4].

Swept-source OCT (SS-OCT) is the latest milestone in retinal and choroidal imaging. To overcome scattering by the RPE, which disabled visualization of deeper lying structures, a longer wavelength was adopted for this machine $(1050 \mathrm{~nm}$ vs $840 \mathrm{~nm}$ in SDOCT).
The scan speed in swept-source instruments is twice that of SD-OCT devices (100 $000 \mathrm{~A}$-scans/sec compared with $50000 \mathrm{~A}$-scans/sec), and more accurate 3-D imaging of the vitreous, retina, and choroid. Wide scans make it possible to present the optic nerve and macula on the same scan [5].

The aim of the current study was to evaluate the potential changes in the subfoveal choroidal thickness (SFCT) after cataract surgery, using Enhanced Depth Imaging OCT.

\section{Patient and Method}

This study was conducted as a prospective cross sectional observational study. Thirty eyes of thirty patients were chosen. Patients were recruited from the outpatient clinic of Nour el Ein hospital in a period from May 2019 to December 2019. A written informed consent was obtained from all patients who participated in the study.

The 30 eyes in this study were subdivided into 2 groups: Group A (15 eyes) - underwent cataract surgery without using non steroidal anti-inflammatory eye drop before surgery. Group B (15 eyes) - underwent cataract surgery with the use of non steroidal anti-inflammatory eye drop 3 times ( one drop every 20 mins for hour ) just before surgery and screened to meet the following inclusion criteria: cataracts with clear OCT data.

\subsection{Inclusion criteria}

1. Patients with cataract with a spherical equivalent (SE) refractive error between -6.00 diopters (D) and $+6.00 \mathrm{D}$ (visually significant cataract) that can provide clear specteral-domain OCT data.

2. Clear cornea.

3. Good pupillary reaction.

4. Uneventful phacoemulsification. 


\subsection{Exclusion criteria}

The following criteria were excluded during patients selection:

1.Patients with dense cataract obscuring the fundus details.

2. Eyes with high myopic, astigmatic error or irregular astigmatism.

3. Eyes with ocular pathology such as corneal opacities, pseudo exfoliation syndrome, ocular hypertension, uveitis, diabetic retinopathy or any other disease affect retina.

4. Eyes with history of intraocular surgery.

5. Eyes with history of ocular trauma.

\subsection{Pre-Operative examination}

- Best corrected visual acuity (BCVA).

- Slit lamp examination of anterior segment.

- IOP measurement by the Goldmann applanation tonometer.

- Posterior segment examination using + 90 diopters lens for slit lamp fundus biomicroscopy.

\subsection{OCT examination}

Images were obtained preoperatively using Spectralis SD-OCT with EDI modality (Heidelberg Engineering, Heidelberg, Germany) for measuring choroidal thickness, subfoveal choroidal thickness and retinal thickness.

The patient pupils were dilated by tropicamide eye drops then the patients were asked to fix on an internal target, and the operator has centered the macular scans.

The measurements of retinal and choroidal thickness were performed using the calipers provided by the Spectralis Heidelberg software on the center of the fovea and $500 \mu \mathrm{m}, 1,000 \mu \mathrm{m}$ away from the fovea in the cardinal directions (vertical, horizontal, nasal, and temporal). A mean of these five values was calculated in order to obtain mean retinal and choroidal thicknesses.

Single $9 \mathrm{~mm}$ horizontal EDI OCT line scan passing through the center of the fovea was used for analysis. The foveal center was defined as the point of maximum depression within an area of $500 \mu \mathrm{m}$ in diameter.

The choroid was measured from the outer portion of the hyper reflective line running from the retinal pigment epithelium to the chorio-scleral interface.

The subfoveal choroidal thickness was measured by the manual caliper function in the Heidelberg Spectralis OCT software (Heidelberg Engineering, Heidelberg, Germany).

The retinal thickness was measured from the inner limiting membrane to the outer boundary of the retinal pigment epithelium.

\subsection{Post-Operative OCT examination}

Images were obtained one week postoperatively, one month postoperatively and three months postoperatively.

The resultant images were viewed and measured with Heidelberg Eye Explorer software (Heidelberg Engineering, Heidelberg, Germany).
Three scans were obtained for each tested eye and the mean of these scans was used for the analysis. The Heidelberg Spectralis OCT software measured the thickness and calculated average thickness.

Data were collected and entered to the computer using SPSS (Statistical Package for Social Science) program for statistical analysis (Chicago,Inc, Illinose, program for statistical analysis) version 18.

\section{Results}

The current study shows that $50 \%$ of our patients were males and $50 \%$ were females. The mean of the age was $44.1 \pm 7.9$ years.

The current study shows that $56.7 \%$ of the eyes were OD and $43.3 \%$ were OS. The anterior chamber, pupil and the fundus were normal in all the patients. The predominant type of cataract was N II + PSC(26.7\%) followed by $\mathrm{N}$ III + PSCandvisually significant PSC $(16.7 \%$ each). The mean of the IOP was $14.5 \pm 1.8$ $\mathrm{mmHg}$.

The current study shows that there was no statistical significant difference between Mean Choroidal Thickness (MCT) readings pre-operative,

1 week, 1 month and 3 months post-operative of the studied group $(\mathrm{P}>0.05)$.

The current study shows that there was no statistical significant difference between SFCT readings preoperative, 1 week, 1 month and 3 months post-operative of the studied group $(\mathrm{P}>0.05)$.

The current study show statistical significant difference between MMT readings pre-operative and 1 month post-operative of the studied group $(\mathrm{P}<0.05)$.

The current study shows that there was no statistical significant difference between both groups as regard the MCT, SFCT and MMT pre-operative, 1 week, 1 month and 3 months post-operative $(\mathrm{P}>0.05)$.

The current study shows that there was statistical significant difference in the MCT, SFCT and MMT preoperative and both 1 week and 1 month post-operative in group $\mathrm{A}(\mathrm{P} \leq 0.05)$. While there was no statistical significant difference between pre-operative and 3 months post-operative $(\mathrm{P}>0.05)$.

The current study shows that there was statistical significant difference in the MCT, SFCT and MMT between 1 and 3 months post-operative in group $\mathrm{A}(\mathrm{P} \leq$ 0.05 ). In addition, there was statistical significant difference between $1 \mathrm{w}$ and 1 months post-operativeas regard the MMT and between $1 \mathrm{w}$ and 3 months posroperative as regard both the MCT and SFCT $(\mathrm{P} \leq 0.05)$.

The current study shows that there was statistical significant difference in the MCT, SFCT and MMT preoperative and both 1 week and 1 month post-operative in group $\mathrm{B}(\mathrm{P} \leq 0.05)$. While there was no statistical significant difference between pre-operative and 3 months post-operative except for the MMT $(\mathrm{P}>0.05)$.

The current study shows that there was statistical significant difference between both 1 week \& 1 month and 1 month \& 3 months post-operative as regard the MCT\& SFCT $(\mathrm{P} \leq 0.05)$. 
The current study shows that there was difference in MCT, SFCT and MMT changes between both groups while the changes had no statistical significant difference except for the MMT measures pre-op \& 3 months post-operative and 1 months \& 3 months postoperative $(\mathrm{P} \leq 0.05)$.

Table (1) Comparison of the MCT, SFCT and MMT pre-operative and 1 week, 1 month and 3 months post-operative in group A.

\begin{tabular}{|c|c|c|c|c|c|c|c|}
\hline & \multicolumn{4}{|c|}{$\begin{array}{c}\text { Group A } \\
N=15\end{array}$} & \multicolumn{3}{|c|}{ Paired sample t-test } \\
\hline & $\begin{array}{c}\text { Pre- } \\
\text { operative } \\
\text { Mean } \pm \text { SD }\end{array}$ & $\begin{array}{c}\text { 1w post- } \\
\text { operative } \\
\text { Mean } \pm \text { SD }\end{array}$ & $\begin{array}{c}\text { 1m post-operative } \\
\text { Mean } \pm \text { SD }\end{array}$ & $\begin{array}{c}\text { 3m post-operative } \\
\text { Mean } \pm \text { SD }\end{array}$ & $\mathbf{P 1}$ & $\mathbf{P 2}$ & P3 \\
\hline MCT & $200 \pm 84$ & $232 \pm 73$ & $230 \pm 75$ & $196 \pm 60$ & 0.001 & 0.001 & 0.169 \\
\hline SFCT & $211 \pm 93$ & $244 \pm 85$ & $241 \pm 83$ & $213 \pm 79$ & 0.005 & 0.002 & 0.455 \\
\hline MMT & $279 \pm 14$ & $288 \pm 15$ & $302 \pm 18$ & $282 \pm 5$ & 0.025 & 0.001 & 0.185 \\
\hline
\end{tabular}

P1 (significance between pre-operative and 1w postoperative reading).

P2 (significance between pre-operative and 1 month post-operative reading).

Table (2) Comparison of the MCT, SFCT and MMT pre-operative and 1 week, 1 month and 3 months post-operative in group B.

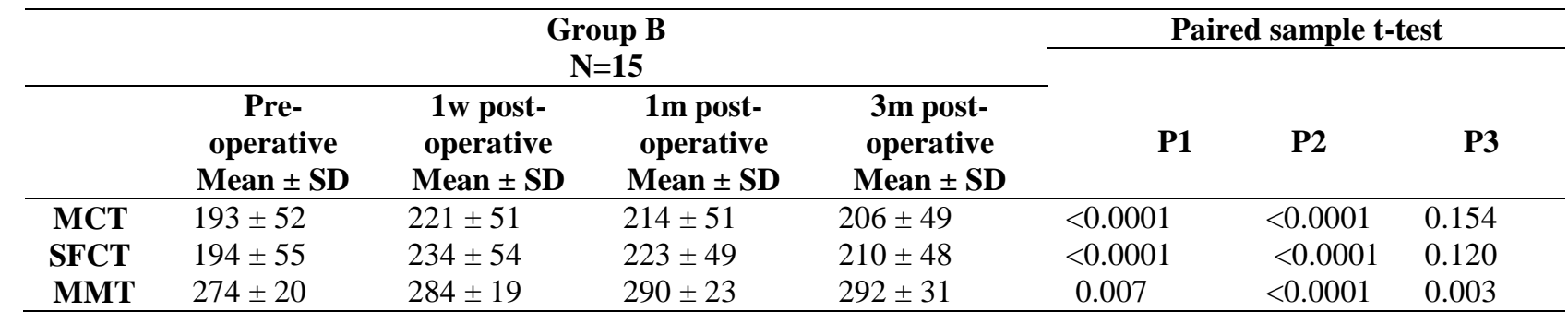

P1 (significance between pre-operative and 1w postoperative reading).

P2 (significance between pre-operative and 1month

P3 (no significance between pre-operative and 3 months post-operative reading). post-operative reading).

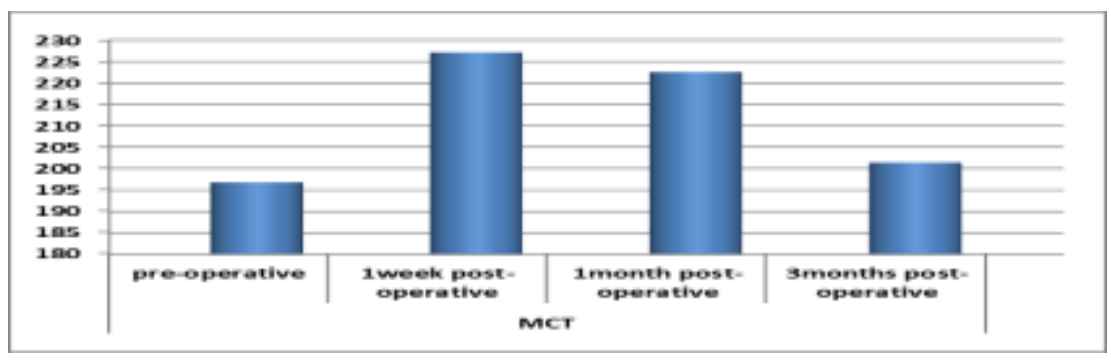

Graph (1) Bar chart showing the MCT pre-operative, 1 week, 1 month and 3 months post-operative of the studied group.

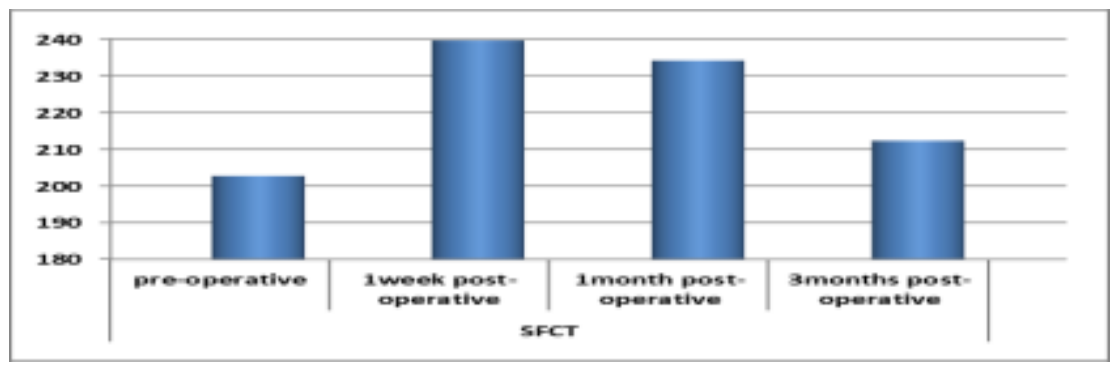

Graph (2) Bar chart showing the SFCT pre-operative, 1 week, 1 month and 3 months post-operative of the studied group 


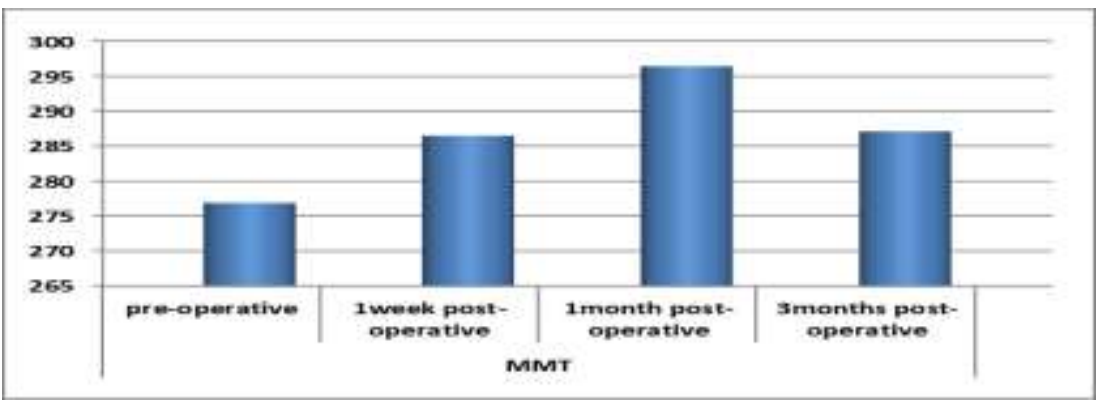

Graph (3) Bar chart showing the MMT pre-operative, 1 week, 1 month and 3 months post-operative of the studied group
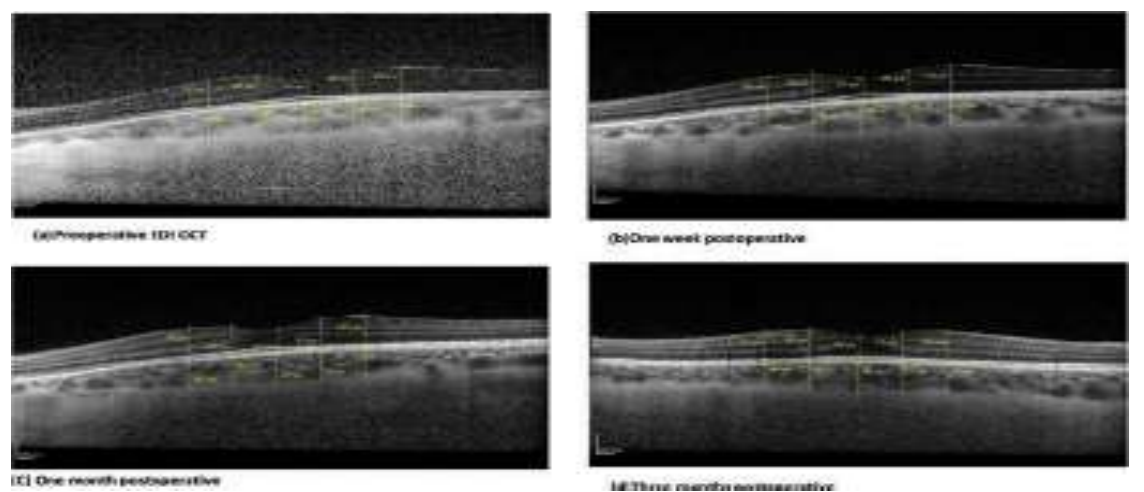

Fig (1) (a) Preoperatively, (b) 1 week postoperatively, (c) 1 month postoperatively, and (d) 3 months postoperatively showing the serial OCTs of a case demonstrating changes in thickness over time.

\section{Discussion}

Phacoemulsification seems to be able to induce morphologic changes in the choroid layers. $\mathrm{Xu}$ et al., 2011 [6] Have disclosed interesting results on the effect of cataract surgery in the choroid. They have investigated the expression of genes that might affect the blood-retinal barriers, including cytokine IL-1b, chemokines CCL2 and SDF-1, and growth factors FGF and VEGF, in the retina and the RPE/choroid of mice undergoing extra capsular lens extraction. They found that the expression of these genes was markedly up regulated in the retina and in the choroid. However, the level of up regulation was less elevated in the $\mathrm{RPE} /$ choroid than in the retina and was delayed in the $\mathrm{RPE} /$ choroid, occurring $24 \mathrm{~h}$ post-operation versus 30 min in the retina. $\mathrm{Xu}$ et al., 2011 [6] also found that the protein IL-1b, which is a pro-inflammatory cytokine, was strongly detected in the ganglion cell layer, inner cell layer, and in the choroid of operated mice eyes.

However, how cataract surgery induces retinal and choroidal inflammation is not understood. It is known that the surgical trauma induces releases of prostaglandins in the aqueous humor that causes a disruption of the blood-aqueous barrier. This results in the accumulation of other inflammatory mediators such as endotoxin, immune complex, and cytokines in the aqueous humor.

These inflammatory mediators diffuse into the vitreous cavity to reach the retina, where they are responsible for a rupture of the inner blood-retinal barrier resulting in another cascade of inflammatory mediators secretion together with an increased permeability from the perifoveal Capillaries [7].

The outer blood-retinal barrier has also been shown to be disrupted as a consequence of post-cataract surgery inflammation. Thus, the inflammatory response of the anterior segment would lead to the occurrence of a posterior segment inflammation. Another explanation is that the surgical traumatism induces an inflammatory response with cytokine gene expression in all the structures of the eye at the same time, which would account for the acute (since $30 \mathrm{~min}$ after surgery) inflammatory gene transcription observed in the retina after cataract surgery [6].

In our study, we found that the mean macular thickness was $276.9 \pm 17.6 \mathrm{um}$. It significantly increased to $286.5 \pm 17.6 \mathrm{um}$ at one week post operatively, then it increased to $296.4 \pm 21.4 \mathrm{um}$ at one month post operatively, and reached to $287.1 \pm 22.1 \mathrm{um}$ at three months post operatively.

This results is in agreement with Pierru et al., 2014 [8] and Falcao et al., 2014[9]. Pierru et al., 2014 reported that before surgery, the mean central macular (CMT) thickness was $233.8 \pm 48$ um for all eyes. It significantly increased at 1 month and 3 months after phacoemulsification to $251.8 \pm 78$ um and $247.6 \pm 81$ um respectively. While Falcao et al., 2014 found that before surgery, mean retinal thickness was $308.77 \pm 14.69 \mu \mathrm{m}$ in vertical scans and 304.49 \pm 15.37 $\mu \mathrm{m}$ in horizontal scans. After cataract surgery, there was a statistically significant increase in mean macular 
thickness one week after surgery and an even greater increase one month after surgery.

One hypothesis would be a post inflammatory rupture in the outer blood-retinal membrane, which in association with the rupture of the inner blood-retinal barrier, would enhance intraretinal accumulation of fluid. Experimental studies have suggested that the outer blood-retinal membrane plays a role in the pathogenesis of macular edema. A disruption of the outer bloodretinal membrane would lead to abnormal inflow of fluid into the retina resulting in accumulation of fluid in the retinal layers [10].

In other pathologies, it is believed that the choroid is primarily affected by the disease and leads to secondary retinal manifestations such as subretinal edema or RPE leaks. In serous central chorioretinopathy or polypoidal choroidal vasculopathy [11]. The choroidal thickening precedes the occurrence of retinal abnormalities. In these pathologies, the increased choroidal thickness is associated with a choroidal vascular hyperpermeability, generally arising from the choriocapillaris [12]. In our study, angiographies were not performed in PCME patients, which enabled us to see whether the increased choroidal thickness was associated with an increased choroidal hyperpermeability.

Pierru et al., 2014[8] shown that the subfoveal choroidal thickness increased after cataract surgery despite prophylactic anti-inflammatory eye drops, showing that cataract surgery may induces inflammatory changes within the choroid. To our knowledge, which is in agreement with correct study is the second report of an increase of subfoveal choroidal thickness in patients prior to the development of PCME.

According to the current study, as regard NSAID, the patients were divided into 2 groups: group A without NSAID and group B with NSAID as there was no statistical significant difference between both groups as reguard the MCT, SFCT and MMT pre-operative, 1 week, 1 month and 3 months post-operative $(\mathrm{P}>0.05)$.

Donnenfeld et al., 2006 [13] chooses to start NSAIDs 3 days before surgery, but noted that even beginning these 1 day beforehand can make a big difference, also noted that Treating patients the day of surgery doesn't give sufficient anti-inflammatory effects, so he start NSAIDs preoperatively. The study get a very good effect with 1 day and a little bit better effect with 3 days.

The study of Kessel et al., 2014 [14] suggested using topical NSAIDs preoperative to control the postoperative inflammation and to prevent CME after routine phacoemulsification surgery. They found that the group used topical NSAIDs preoperatively are sucssed in reducing CME than the one non used.

However, the relationship between the extent of choroidal thickness and the development of PCME needs to be clarified. Further studies are needed to investigate if the choroid plays a role in PCME physiopathology.

\section{Conclusion}

There was no significant difference in B-group which administered NSAIDs before surgery. Uncomplicated phacoemulsification induces nonpathologic increases in retinal macular thickness probably due to the inflammatory insult of the surgery; however these changes are not accompanied by significant changes in choroidal thickness.

\section{References}

[1] P.Taravati, DL.Lam, T.Leveque and Van RN.Gelder. Postcataract surgical inflammation. Curr Opin Ophthalmol.vol.23,pp.12-18,2012.

[2] Y.Yonekawa and IK.Kim. Pseudophakic cystoid macular edema. Curr Opin Ophthalmol.vol.23,pp.26-32,2012.

[3] JF.Arevalo, AF.Lasave and JD.Arias. Clinical applications of optical coherence tomography in the posterior pole: the 2011 José Manuel Espino Lecture - Part II. Clinical Ophthalmology (Auckland, NZ).vol.7,pp.2181-2206, 2013.

[4] RF.Spaide, H.Koizumi and MC.Pozonni. Enhanced depth imaging spectral-domain optical coherence tomography. Am J Ophthalmol.vol.146,pp.496-500,2008.

[5] Z.Michalewska, J.Michalewski and J.Nawrocki. Going deeper and going wider. Retinal Physician.vol.3,pp.42-48,2013.

[6] H.Xu, M.Chen, JV.Forrester and N.Lois. Cataract surgery induces retinal pro-inflammatory gene expression and protein secretion. Invest Ophthalmol Vis Sci.vol.52,pp.249-255,2011.

[7] K.Miyake, N.Ibaraki. Prostaglandins and cystoid macular edema. Survey Ophthalmol.vol.47,pp.S203-S218,2002.

[8] A.Pierru, M.Carles, P.Gastaud and S.Baillif. Measurement of subfoveal choroidal thickness after cataract surgery in enhanced depth imaging optical coherence tomography. Invest Ophthalmol Vis Sci.vol.55,pp.4967-4974. 14-14172,2014.

[9] MS.Falcao, NM.Gon ${ }_{s}$ calvez and P.Freitas-Costa. Choroidal and macular thickness changes induced by cataract surgery. Clin Ophthalmol.vol.8,pp.5560,2014.

[10]N.Bhagat, RA.Grigorian, A.Tutela and MA.Zarbin. Diabetic macular edema: pathogenesis and treatment. Surv Ophthalmol.vol.54,pp.132,2009.

[11]RF.Spaide, M.Goldbaum and DW.Wong. Serous detachment of the retina. Retina.vol.23,pp.820$846,2003$.

[12] I.Maruko, T.Lida, H.Oyamada, Y.Sugano, A.Ojima, T.Sekiryu. Choroidal thickness changes after intravitreal ranibizumab and photodynamic therapy in recurrent polypoidal choroidal vasculopathy. Am J Ophthalmol.vol.156,pp.548$556,2013$.

[13] ED.Donnenfeld, HD.Perry, JR.Wittpenn, R.Solomon, A.Nattis and T.Chou. Preoperative ketorolac tromethamine $0.4 \%$ in 
phacoemulsification outcomes: pharmacokineticresponse curve. J Cataract Refract Surg.vol.32(9),pp.147482, 2006.

[14] L.Kessel, B.Tendal, KJ.Jørgensen, D.Erngaard, P.Flesner, JL.Andresen. Post-cataract prevention of inflammation and macular edema by steroid and nonsteroidal anti-inflammatory eye drops: a systematic review. Ophthalmology.vol121(10),pp.1915-1924,2014. 